\title{
Evolutionary Computing
}

\author{
A.E. Eiben ${ }^{a}$ and M. Schoenauer ${ }^{b}$ \\ ${ }^{a}$ Free University Amsterdam - The Netherlands \\ http://www.cs.vu.nl/ gusz/ \\ ${ }^{\mathrm{b}}$ INRIA Rocquencourt - France \\ http://www-rocq.inria.fr/fractales/Staff/Schoenauer
}

Evolutionary computing (EC) is an exciting development in Computer Science. It amounts to building, applying and studying algorithms based on the Darwinian principles of natural selection. In this paper we briefly introduce the main concepts behind evolutionary computing. We present the main components all evolutionary algorithms (EA), sketch the differences between different types of EAs and survey application areas ranging from optimization, modeling and simulation to entertainment.

\section{Introduction}

Surprisingly enough, the idea to apply Darwinian principles to automated problem solving originates from the fifties, long before the breakthrough of computers [9]. During the sixties three different implementations of this idea have been developed at three different places. In the USA Fogel introduced evolutionary programming [118, while Holland called his method a genetic algorithm 1312. In Germany Rechenberg and Schwefel invented evolution strategies [1516]. For about 15 years these areas developed separately; it is since the early nineties that they are envisioned as different representatives ("dialects") of one technology, called evolutionary computing. It was also in the early nineties that a fourth stream following the general ideas has emerged - genetic programming [142]. The contemporary terminology denotes the whole field by evolutionary computing and considers evolutionary programming, evolution strategies, genetic algorithms, and genetic programming as sub-areas.

\section{What is an evolutionary algorithm?}

The common underlying idea behind all these techniques is the same: given a population of individuals, the environmental pressure causes natural selection (survival of the fittest) and hereby the fitness of the population is growing. It is easy to see such a process as optimization. Given an objective function to be maximized we can randomly create a set of candidate solutions and use the objective function as an abstract fitness measure (the higher the better). Based on this fitness, some of the better candidates are chosen to seed the next generation by applying recombination and/or mutation. Recombination is applied to two selected candidates, the so-called parents, and results one or two new candidates, the children. Mutation is applied to one candidate and results in one new candidate. Applying recombination and mutation leads to a set of new candidates, the offspring. Based on their fitness these offspring compete with the old candidates for a place in the next generation. This process can be iterated until a solution is found or a previously set time limit is reached. Let us note that many components of such an evolutionary process are stochastic. According to Darwin, the emergence of new species, adapted to their environment, is a consequence of the interaction between the survival of the fittest mechanism and undirected variations. Variation operators must be stochastic, the choice on which pieces of information will be exchanged during recombination, as well as the changes in a candidate solution during mutation, are random. 
On the other hand, selection operators can be either deterministic, or stochastic. In the latter case fitter individuals have a higher chance to be selected than less fit ones, but typically even the weak individuals have a chance to become a parent or to survive. The general scheme of an evolutionary algorithm can be given as follows.

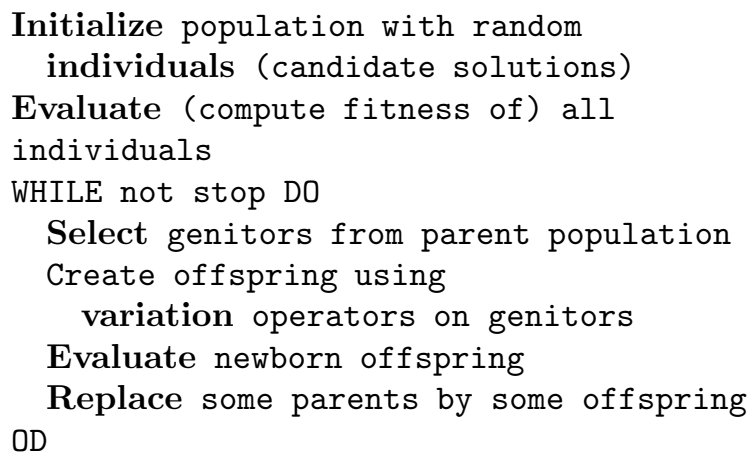

Let us note that this scheme falls in the category of generate-and-test, also known as trialand-error, algorithms. The fitness function represents a heuristic estimation of solution quality and the search process is driven by the variation operators (recombination and mutation creating new candidate solutions) and the selection operators. Evolutionary algorithms (EA) are distinguished within in the family of generate-and-test methods by being population based, i.e. process a whole set of candidate solutions and by the use of recombination to mix information of two candidate solutions.

The aforementioned "dialects" of evolutionary computing follow the above general outlines and differ only in technical details.

\section{Critical issues}

There are some issues that one should keep in mind when designing and running an evolutionary algorithm. These considerations concern all of the "dialects", and will be discussed here in general, without a specific type of evolutionary algorithm in mind.

One crucial issue when running an EA is to try to preserve the genetic diversity of the population as long as possible. Opposite to many other optimization methods, EAs use a whole population of individuals - and this is one of the reasons for their power. However, if that populations starts to concentrate in a very narrow region of the search space, all advantages of handling many different individuals vanish, while the burden of computing their fitnesses remains. This phenomenon is known as premature convergence. There are two main directions to prevent this: a priori ensuring creation of new material, for instance by using a high level of mutation (see section 4.3.3); or a posteriori manipulating the fitnesses of all individuals to create a bias against being similar, or close to, existing candidates. A well-known technique is the so-called niching mechanism.

Exploration and exploitation are two terms often used in EC. Although crisp definitions are lacking [7] there has been a lot of discussion about them. The dilemma within an optimization procedure is whether to search around the best-so-far solutions (as their neighborhood hopefully contains even better points) or explore some totally different regions of the search space (as the bestso-far solutions might only be local optima). An EA must be set up in such a way that it solves this dilemma without a priori knowledge of the kind of landscape it will have to explore. The exploitation phase can sometimes be "delegated" to some local optimization procedure, whether called as a mutation operator, or systematically applied to all newborn individuals, moving them to the nearest local optimum. In the latter case, the resulting hybrid algorithm is called a memetic algorithm.

In general, there are two driving forces behind an EA: selection and variation. The first one represents a push toward quality and is reducing the genetic diversity of the population. The second one, implemented by recombination and mutation operators, represents a push toward novelty and is increasing genetic diversity. To have an EA work properly, an appropriate balance between these two forces has to be maintained. At the moment, however, there is not much theory supporting practical EA design. 


\section{Components of evolutionary algorithms}

\subsection{Representation}

Solving a given problem with an EA starts with specifying a representation of the candidate solutions. Such candidate solutions are seen as phenotypes that can have very complex structures. Applying variation operators directly to these structures might not be possible, or easy. Therefore these phenotypes are represented by corresponding genotypes. The standard EC machinery consists of many off-the-shelf variation operators acting on a specific genotype space, for instance bit-strings, real-valued vectors, permutations of integers, or trees. Designing an EA thus often amounts to choosing one of the standard representations with the corresponding variation operators in mind. However, one strength of EAs is their ability to tackle any search space provided that initialization and variation operators are available. Choosing a standard option is, therefore, not necessary.

\subsection{Fitness or evaluation function}

Fitness-based selection is the force that represents the drive toward quality improvements in an EA. Designing the fitness function (or evaluation function) is therefore crucial.

The first important feature about fitness computation is that it represents $99 \%$ of the total computational cost of evolution in most realworld problems. Second, the fitness function very often is the only information about the problem in the algorithm: Any available and usable knowledge about the problem domain should be used.

\subsection{Representation dependent \\ 4.3.1. Initialization}

The initial population is usually created by some random sampling of the search space, generally performed as uniformly as possible. However, in some cases, uniform sampling might not be well-defined, e.g. on parse-tree spaces, or on unbounded intervals for floating-point numbers.

A common practice also is to inoculate some known good solutions into the initial population. But beware that no bias is better than a wrong bias!

\subsubsection{Crossover}

Crossover operators take two (or more) parents and generate offspring by exchange of information between the parents. The underlying idea to explain crossover performance is that the good fitness of the parents is somehow due to precise parts of their genetic material (termed building blocks), and the recombining those building blocks will result in an increase in fitness.

Nevertheless, there are numerous other ways to perform crossover. For instance, crossing over two vectors of floating-points values can be done by linear combination (with uniformly drawn weights) of the parents values. The idea is that information pertaining to the problem at hand should be somehow exchanged.

Note that the effect of crossover varies from exploration when the population is highly diversified to exploitation when it starts to collapse into a small region of the search space.

\subsubsection{Mutation}

Mutation operators are stochastic transformations of an individual. The usual compromise between exploration and exploitation must be maintained: large mutations are necessary from theoretical reasons (it ensures the ergodicity of the underlying stochastic process), that translate practically (it is the only way to reintroduce genetic diversity in the end of evolution); but of course too much too large mutation transform the algorithm into a random walk - so most mutations should generate offspring close to their parents. There is no standard general mutation, but general trends are to modify the value of a component of the genotype with a small probability (e.g. flip one bit of a bitstring, or, in case of real-valued components, add zero-mean Gaussian noise with carefully tuned standard deviation).

\subsubsection{The historical debate}

There has long been a strong debate about the usefulness of crossover. The GA community considers crossover to be the essential variation operator 1312, while mutation is only a background necessity. On the other hand, the historical ES 15 16] and EP 11] researchers did not use any crossover at all, and even claimed later that it 
could be harmful 10.

The general agreement nowadays is that the answer is problem-dependent: If there exists a "semantically meaningful" crossover for the problem at hand, it is probably a good idea to use it. But otherwise mutation alone might be sufficient to find good solutions - and the resulting algorithm can still be called an Evolutionary Algorithm.

\subsection{Representation-independent}

\subsubsection{Artificial Darwinism}

Darwin's theory states that the fittest individuals reproduce and survive. The evolution engine, i.e. the two steps of selection (of some parents to become genitors) and replacement (of some parents by newborn offspring) are the artificial implementations of these two selective processes. They differ in an essential way: during selection step, the same parent can be selected many times; during replacement step, each individual (among parents and offspring) either is selected, or disappears for ever.

Proportional selection (aka roulette-wheel) has long been the most popular selection operator: each parent has a probability to be selected that is proportional to its fitness. However, the difficulty is to scale the fitness to tune the selection pressure: even the greatest care will not prevent some super-individual to take over the population in a very short time. Hence the most widely used today is tournament selection: to select one individual, $T$ individuals are uniformly chosen, and the best of these $T$ is returned. Of course, both roulette-wheel and tournament repeatedly act on the same current population, to allow for multiple selection of the very best individuals.

There are two broad categories of replacement methods: either the parents and the offspring "fight" for survival, or only some offspring are allowed to survive. Denoting by $\mu$ (resp. $\lambda$ ) the number of parents (resp. offspring) as in ES history (section 5.2), the former strategy is called $(\mu+\lambda)$ and the latter $(\mu, \lambda)$. When $\mu=\lambda$, the comma strategy is also known as generational replacement: all offspring simply replace all parents. When $\lambda=1$, the (plus!) strategy is then termed steady-state and amounts to choosing one parent to be replaced.
An important point about the evolution engine is the monotonicity of the best fitness along evolution: for instance, ES plus strategies are elitist, i.e. ensure that the best fitness can only increase from one generation to another, while the comma strategies are not elitist - though elitism can be a posteriori added by retaining the best parent when a decrease of fitness is foreseen.

\subsubsection{Termination criterion}

There has been very few theoretical studies about when to stop an Evolutionary Algorithm. The usual stopping criterion is a fixed amount of computing time (or, almost equivalently, of fitness computations). A slightly more subtle criterion is to stop when a user-defined amount of time has passed without improvement of the best fitness in the population.

\subsection{Setting the parameters}

EAs typically have a large number of parameters (e.g. population size, frequency of recombination, mutation step-size, selective pressure, $\ldots)$. The main problem in this respect is that even the individual effect of one parameter is often unpredictable, let alone the combined influence of all parameters. Most authors rely on intensive trials (dozens of independent runs for each possible parameter setting) to calibrate their algorithms - an option that is clearly very time consuming. Another possibility is to use longexisting statistical techniques like ANOVA. A specific evolutionary trend is to let the EA calibrate itself to a given problem, while solving that problem (see section [5.2).

\subsection{Result analysis}

As with any randomized algorithm, the results of a single run of an EA are meaningless. A typical experimental analysis will run say over more than 15 independent runs (everything equal except the initial population), and present averages, standard deviations, and T-test in case of comparative experiments.

However, one should distinguish design problems, where the goal is to find at least one very good solution once, from day-to-day optimization (e.g. control, scheduling,...), where the goal is to consistently find a good solution for different 
inputs. In the design context, a high standard deviation is desirable provided the average result is not too bad. In the optimization context, a good average and a small standard deviation are mandatory.

\section{Historical dialects}

As already quoted, EC arose from independent sources. Of course, each dialect exhibits a large variety in itself; the short descriptions here are necessarily restricted to one or two main variants.

\subsection{Genetic Algorithms}

The standard GA 1312 can be seen as the combination of bit-string representation, with bit-exchange crossover (applied with given probability $p_{c}$ ) and bit-flip mutation (applied to every bit with probability $p_{m}$ ), roulette-wheel selection plus generational replacement (though steadystate replacement can also be used).

Note that other versions of EAs using the same evolution engine with different genotypes (and hence variation operators) are often called GA.

\subsection{Evolution Strategies}

Evolution strategies (ES) [1516 are typically applied to real-valued parameter optimization problems (historically discretized). ES apply to real-valued vectors using Gaussian mutation, no selection and $(\mu+, \lambda)$ replacement strategies. Crossover (historically absent) is performed either by exchanging components, or by doing a linear recombination on some components.

The characteristic feature of ES lies in the selfadaptation of the standard deviation of the Gaussian distribution used in the mutation [1]. The basic idea is to add these parameters to the genotypes, and have them undergo evolution themselves.

\subsection{Evolutionary Programming}

Traditional EP [1] was concerned with evolving finite state automata for machine learning tasks. Representation and operators were specialized for this application area. Each parent gave birth by mutation only to one offspring, and a plus replacement strategy was used to remove half of the individuals. Contemporary EP, however,
8 has evolved to using any representation and different evolution engines, and nowadays differs from ES by using a stochastic form of the plus replacement strategy, and by never using crossover (EP also uses self-adaptation of Gaussian mutation in the case of real-valued genotypes).

\subsection{Genetic Programming}

The youngest brother of the family [142] has a specific application area in machine learning and modeling tasks. A natural representation is that of parse-trees of formal logical expressions describing a model or procedure. Crossover and mutation operators are adapted so that they work on trees (with varying sizes). Evolution engine is "inherited" from GAs (GP has long been seen as GA with tree representation). On the other hand, syntactic expressions - for instance LISP - can be viewed as programs, which makes GP the branch concerned with automatic evolution of programs.

\section{Application areas}

Although it is often stressed that an evolutionary algorithm is not an optimizer in the strict sense 6], optimization problems form the most important application area of EAs. Within this field further distinctions can be made, combinatorial optimization, continuous parameter optimization, or mixed discrete-continuous optimization.

In the framework of combinatorial optimization, it is now recognized that EC alone is not competitive 3] compared to classical Operational Research heuristics. However, hybridization of EC with those specialized OR heuristics gave tremendous results, on benchmark problems (e.g. best-to-date results on some difficult graph coloring, quadratic assignment, or constraint satisfaction instances) as well as on many real-world problems (e.g. time-tabling in universities, crew scheduling in big companies, multiple tours with time-windows in distribution applications, ...). It is worth mentioning here that combinatorial problems is today the most profitable application domain for EC.

When it comes to continuous parametric optimization, the mistake to avoid is to try to 
compete with highly performing numerical methods. However, in many cases such methods do not apply (lack of regularity) or fail (high multimodularity). In such contexts, EC has been successfully used for control, electromagnetism, fluid mechanics, structural analysis, ...

The flexibility of EC allows one to handle representations (section 4.1) that are out of reach of any other method. This is the case for mixed search spaces (with both discrete and continuous variables), and even more for variable length representations (e.g. parse trees of Genetic Programming, see section 5.4). And this opens up the possibility for huge improvements in areas such as Machine Learning (e.g. by evolving sets of rules, cellular automata rules, ... ), modeling (in the general framework of function identification), design and art [4, where restricting the representation of the solutions to a fixed set of parameters definitely bias the search toward poo regions in terms of diversity.

Finally, let us stress that one domain where Evolutionary Algorithms encounter an increasing attention is that of multi-objective optimization: specific selection methods [5] allow one to spread the population of an EA over the Pareto front of a multi-objective problem (the set of the best compromises between the objectives), requiring only a fraction of computing time more than the optimization of a single objective.

\section{Concluding remarks}

Natural evolution can be considered as a powerful problem solver achieving Homo Sapiens from chaos in only a couple of billion years. Computerbased evolutionary processes can also be used as efficient problem solvers for optimization, constraint handling, machine learning and modeling tasks. Furthermore, many real-world phenomena from the study of life, economy, and society can be investigated by simulations based on evolving systems. Last but not least, evolutionary art and design form an emerging field of applications of the Darwinian ideas. We expect that computer applications based on evolutionary principles will gain popularity in the coming years in science, business, and entertainment.

\section{REFERENCES}

1. Th. Bäck and H.-P. Schwefel. An overview of evolutionary algorithms for parameter optimization. Evolutionary Computation, 1(1):1-23, 1993.

2. W. Banzhaf, P. Nordin, R.E. Keller, and F.D. Francone. Genetic Programming - An Introduction. Morgan Kaufmann, 1998.

3. Th. Bäck, D.B. Fogel, and Z. Michalewicz, editors. Handbook of Evolutionary Computation. Oxford University Press, 1997.

4. P. J. Bentley, editor. Evolutionary Design by Computers. Morgan Kaufman, 1999.

5. K. Deb. Multi-Objective Optimization Using Evolutionary Algorithms. John Wiley, 2001.

6. K. A. DeJong. Are genetic algorithms function optimizers? In R. Manner and B. Manderick, editors, Proc. PPSN II, pages 3-13. North Holland, 1992.

7. A.E. Eiben and A. Schippers. On evolutionary exploration and exploitation. Fundamenta Informaticae, 35(1-4):35-50, 1998.

8. D. B. Fogel. Evolutionary Computation. Toward a New Philosophy of Machine Intelligence. IEEE Press, Piscataway, NJ, 1995.

9. D.B. Fogel. Evolutionary Computing: The Fossile Record. IEEE Press, 1998.

10. D.B. Fogel and L.C. Stayton. On the effectiveness of crossover in simulated evolutionary optimization. BioSystems, 32:171-182, 1994.

11. L. J. Fogel, A. J. Owens, and M. J. Walsh. Artificial Intelligence through Simulated Evolution. New York: John Wiley, 1966.

12. D. E. Goldberg. Genetic algorithms in search, optimization and machine learning. Addison Wesley, 1989.

13. J. H. Holland. Adaptation in natural and artificial systems. University of Michigan Press, Ann Arbor, 1975.

14. J. R. Koza. Genetic Programming: On the Programming of Computers by means of Natural Evolution. MIT Press, Massachusetts, 1992.

15. I. Rechenberg. Evolutionstrategie: Optimierung Technisher Systeme nach Prinzipien des Biologischen Evolution. Fromman-Hozlboog Verlag, Stuttgart, 1973.

16. H.-P. Schwefel. Numerical Optimization of Computer Models. John Wiley \& Sons, New-York, 1981. $1995-2^{\text {nd }}$ edition. 